\title{
Dimensional reduction from five-dimensional gauge theories
}

\author{
Francesco Knechtli* and Antonio Rago ${ }^{\dagger}$ \\ Department of Physics, Bergische Universität Wuppertal \\ Gaussstr. 20 \\ D-42119 Wuppertal, Germany \\ E-mail: knechtli@physik.uni-wuppertal.de
}

We study the phase diagram of five-dimensional SU(2) gauge theories on anisotropic lattices with periodic boundary conditions. We locate a line of first order bulk phase transitions and second order phase transitions related to breaking of the center along one direction. A reduction to four dimensions occurs through compactification of one dimension but not only. By choosing a lattice spacing in the extra dimension larger than in the other dimensions, we find hints that the hyperplanes orthogonal to the extra dimension decouple from each other. Our analysis is based on measurements of Polyakov loops, the static potential extracted from Wilson loops and renormalized couplings defined through the static force and its derivative.

WUB/11-20

XXIX International Symposium on Lattice Field Theory

July 10-16 2011

Squaw Valley, Lake Tahoe, California

\footnotetext{
* Speaker.

†Present address: School of Computing and Mathematics, University of Plymouth, Plymouth PL4 8AA, UK
} 


\section{Introduction}

Our interest in five-dimensional gauge theories is motivated by the models of physics beyond the Standard Model called Gauge-Higgs Unification. When dimensional reduction from five to four dimensions occurs, the five-dimensional components of the gauge field behave as scalar fields which could be identified with the Higgs particle. The physical content of the scalar fields is carried by the Polyakov loops winding along the fifth dimension. In this contribution we concentrate on the possibility of dimensional reduction and the mechanisms underlying it. We emphasize that due to the non-renormalizability (or triviality) of five-dimensional gauge theories, it is important to perform this study in five dimensions, despite the computational cost. In fact triviality is related to the existence of a bulk phase transition in five dimensions [1].

Our setup is a $L_{T} \times L_{\mathrm{S}}^{3} \times L_{5}$ Euclidean lattice. The SU(2) Yang-Mills theory is discretized using the anisotropic Wilson plaquette gauge action [2]

$$
S=\frac{\beta}{2} \sum_{x}\left[\frac{1}{\gamma} \sum_{\mu<v} \operatorname{tr}\{1-U(x ; \mu, v)\}+\gamma \sum_{\mu} \operatorname{tr}\{1-U(x ; \mu, 5)\}\right],
$$

where the traces are over oriented plaquettes and the indices $\mu, v$ run over the usual four dimensions. The lattice couplings $\beta$ and $\gamma$ are are related in the classical limit to the lattice spacings $a_{4}$ in four dimensions, $a_{5}$ in the fifth dimension and to the dimensionful bare gauge coupling $g_{5}$ through

$$
\beta=\frac{4 a_{4}}{g_{5}^{2}} \quad \text { and } \quad \gamma=\frac{a_{4}}{a_{5}} .
$$

An alternative but equivalent parameter pair is

$$
\beta_{4}=\frac{\beta}{\gamma} \quad \text { and } \quad \beta_{5}=\beta \gamma
$$

We take periodic boundary conditions in all the directions. Here we discuss the main results and refer to [4] for more details. In [5] we report on recent results using orbifold boundary conditions.

\section{The mean-field phase diagram}

In $[3,6]$ the five-dimensional $\mathrm{SU}(2)$ gauge theory has been investigated using the meanfield method, which is an expansion around a saddle-point. The saddle-point configuration is parametrized by $\overline{\mathbf{1}} \mathbf{1}$ for the four-dimensional links and $\bar{v}_{5} \mathbf{1}$ for extra dimensional links ( $\mathbf{1}$ is the $2 \times 2$ identity matrix) and is found by iteratively solving the equations

$$
\bar{v}=\frac{I_{2}(\bar{h})}{I_{1}(\bar{h})} \quad \text { and } \quad \bar{v}_{5}=\frac{I_{2}\left(\bar{h}_{5}\right)}{I_{1}\left(\bar{h}_{5}\right)}
$$

where $I_{1,2}$ are modified Bessel functions and $\bar{h}=6 \beta_{4} \bar{v}^{3}+2 \beta_{5} \overline{v v}_{5}^{2}, \bar{h}_{5}=8 \beta_{5} \bar{v}^{2} \bar{v}_{5}$. The result is shown in Fig. 1. There are three phases, one in which $\bar{v} \neq 0, \bar{v}_{5} \neq 0$ (blue area, the deconfined phase), one in which $\bar{v} \neq 0, \bar{v}_{5}=0$ (red area, the layered phase) and one in which $\bar{v}=0=\bar{v}_{5}$ (white area, the confined phase). The black points mark values where no convergence to a finite value is attained and the dashed line represents $\beta_{4}=\beta_{5}$. 


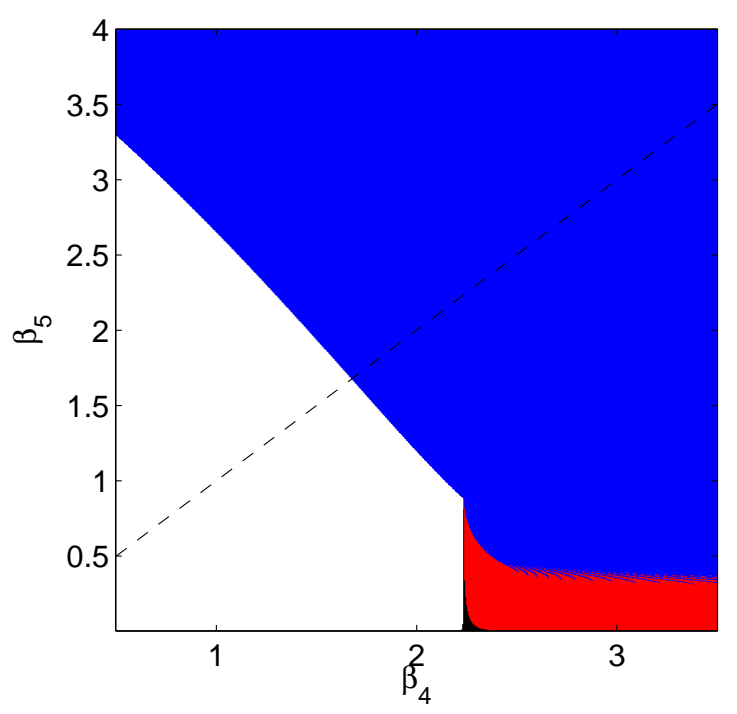

Figure 1: The phase diagram of the five-dimensional SU(2) gauge theory using the anisotropic Wilson plaquette gauge action in the mean-field approximation (saddle-point solution).

In [6] it was found that dimensional reduction occurs when $\beta_{4}>\beta_{5}$, or $a_{5}>a_{4}$. The transition from the deconfined to the layered phase turns second order and a continuum limit can be taken. In the limit, the four-dimensional hyperplanes orthogonal to the fifth dimension decouple and a theory in the universality class of the four-dimensional Ising model is obtained. The correlation length is given by the inverse scalar mass. The continuum limit is taken in finite volume keeping two quantities, the anisotropy $\gamma$ and the ratio of the vector to the scalar mass, fixed. In the following we verify if this scenario is confirmed by Monte Carlo simulations of the full theory.

\section{The phase diagram from Monte Carlo simulations}

\subsection{Bulk phase transitions}

Fig. 2 is the summary of our results concerning the phase diagram [4]. The points marked by red squares (and connected by a grey band to guide the eye) are bulk phase transitions and they are signaled by the behavior of the plaquette. Fig. 3 shows this transition in a scan of $\beta_{5}$ keeping $\beta_{4}=2.33$ fixed. The hysteresis effect is seen provided the four-dimensional volume is large enough, in this case we need $L_{T}=L_{\mathrm{s}} \geq 14\left(L_{5}\right.$ is approximately $\left.L_{\mathrm{s}} / 2\right)$. If the volume is smaller the transition appears like a cross-over, due to compactification of these directions (we will return to this issue in the next section). In order to study bulk phase transitions when $\beta_{4}>\beta_{5}$ we need to ensure that four directions of the lattice $\left(L_{T}, L_{\mathrm{S}}\right)$ are large enough, since $a_{4}<a_{5}$. This has to be compared with studies at $\beta_{5}>\beta_{4}$, where $L_{5}$ only has to be made sufficiently large. In summary, the grey band in Fig. 2 is a line of first order phase transitions. From measurements of the static potential we know that this line separates the confined phase at smaller $\beta_{4}$ from the deconfined phase. 


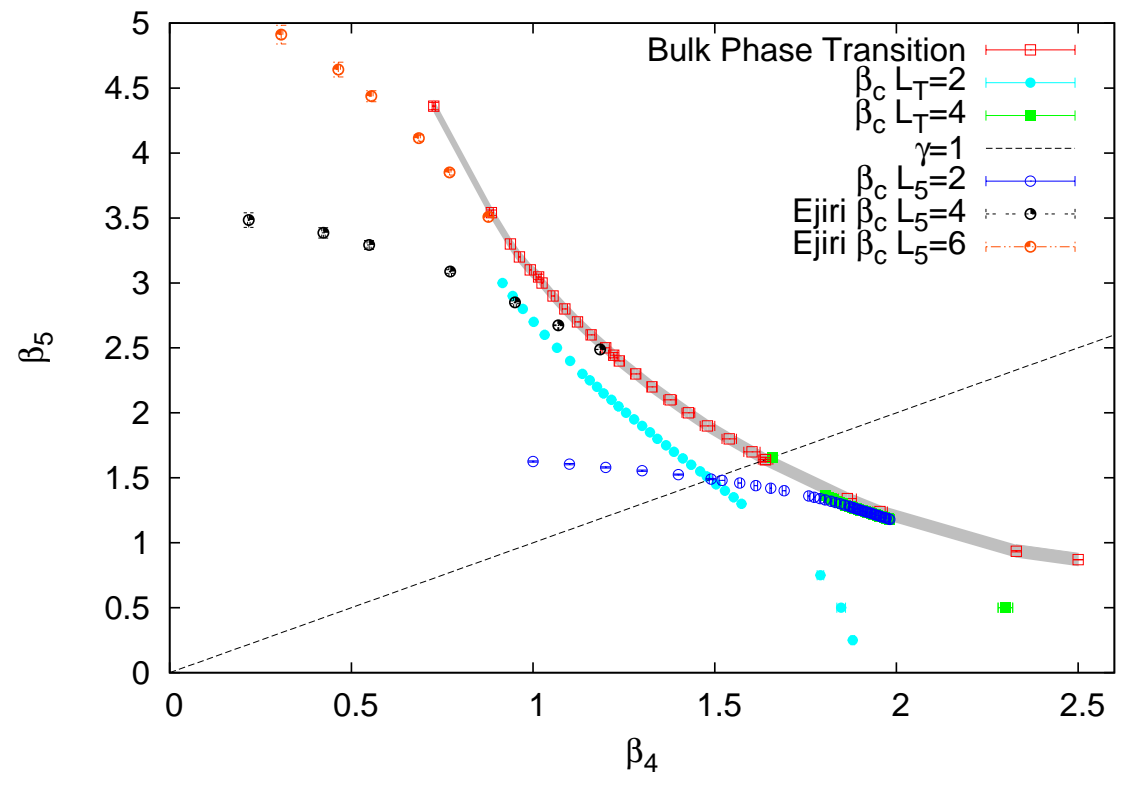

Figure 2: The phase diagram of the five-dimensional SU(2) gauge theory simulated with the anisotropic Wilson plaquette gauge action [4].

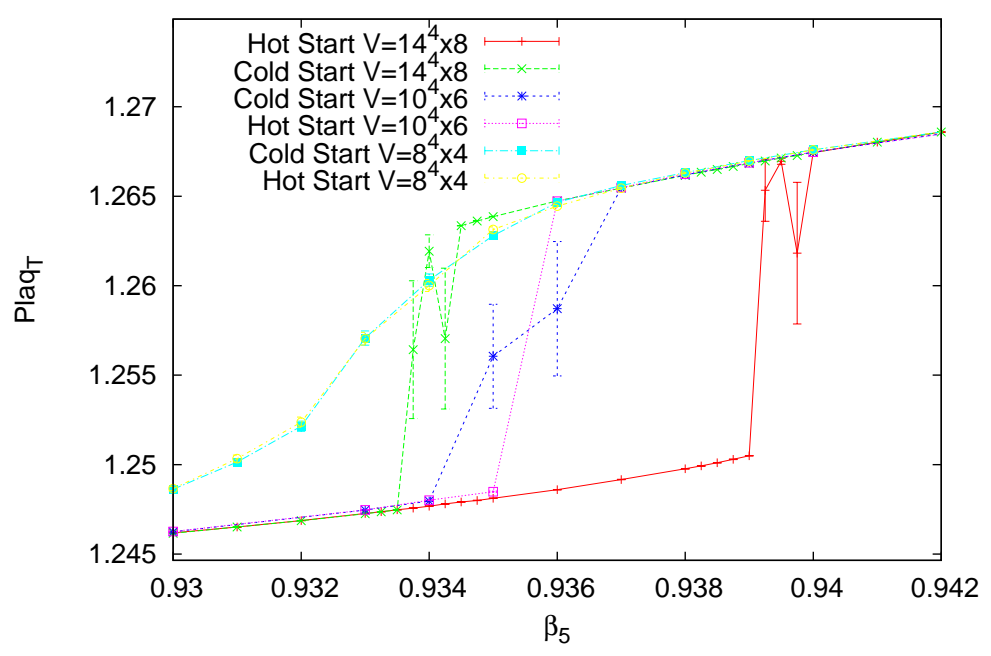

Figure 3: Bulk phase transition at $\beta_{4}=2.33$. Lattices with $L_{\mathrm{s}} \geq 14$ are needed to see the hysteresis in the plaquette (here $\mathrm{Plaq}_{T}$ is the four-dimensional plaquette).

\subsection{Phase transitions related to center breaking}

Fig. 2 presents phase transition points due to compactification of one direction, which are signaled by the behavior of the Polyakov loop winding along the small direction. When compactification occurs, the Polyakov loop expectation value (its absolute value) becomes non-zero. At $\beta_{5}>\beta_{4}$ there are second order phase transitions when $L_{5}=2,4,6, \ldots$ which have been studied in $[7,8]$. Our new results mainly concern the region $\beta_{4}>\beta_{5}$, where we compactify $L_{T}=2,4, \ldots$. Fig. 4 shows a study of the transition with $L_{T}=2$ at $\beta_{5}=0.5$. We do a finite size scaling analysis 

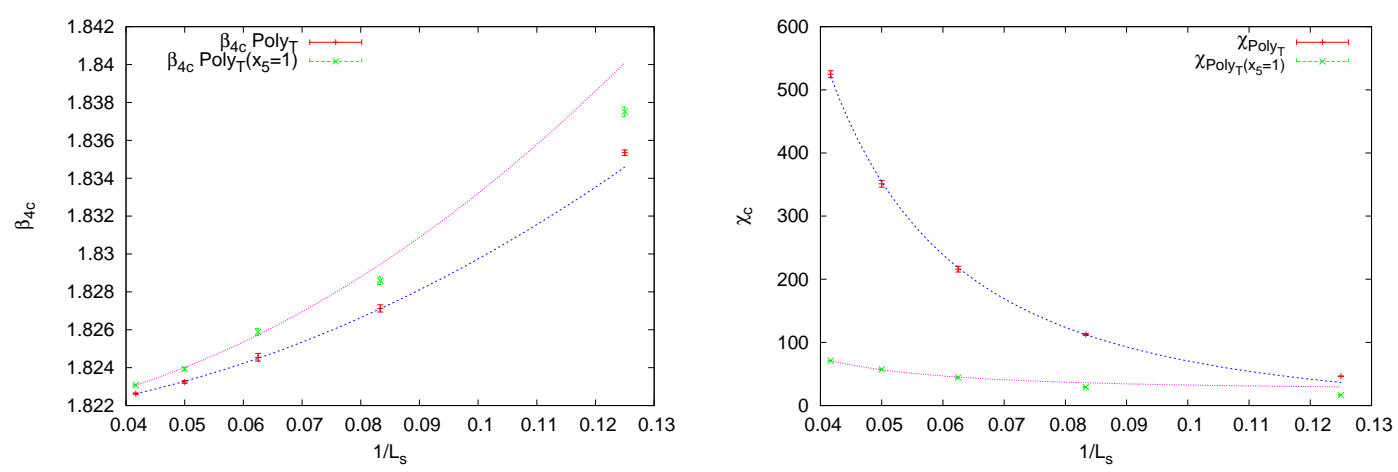

Figure 4: Finite size scaling analysis for the transition at $\beta_{5}=0.5, L_{T}=2$ : the critical coupling $\beta_{4 c}$ (left plot) and the critical susceptibility $\chi_{c}$ of the temporal Polyakov loop (right plot). The lines are fits to the data using the critical exponents of the four-dimensional Ising model. Two definitions of the Polyakov loop are used, see [4].
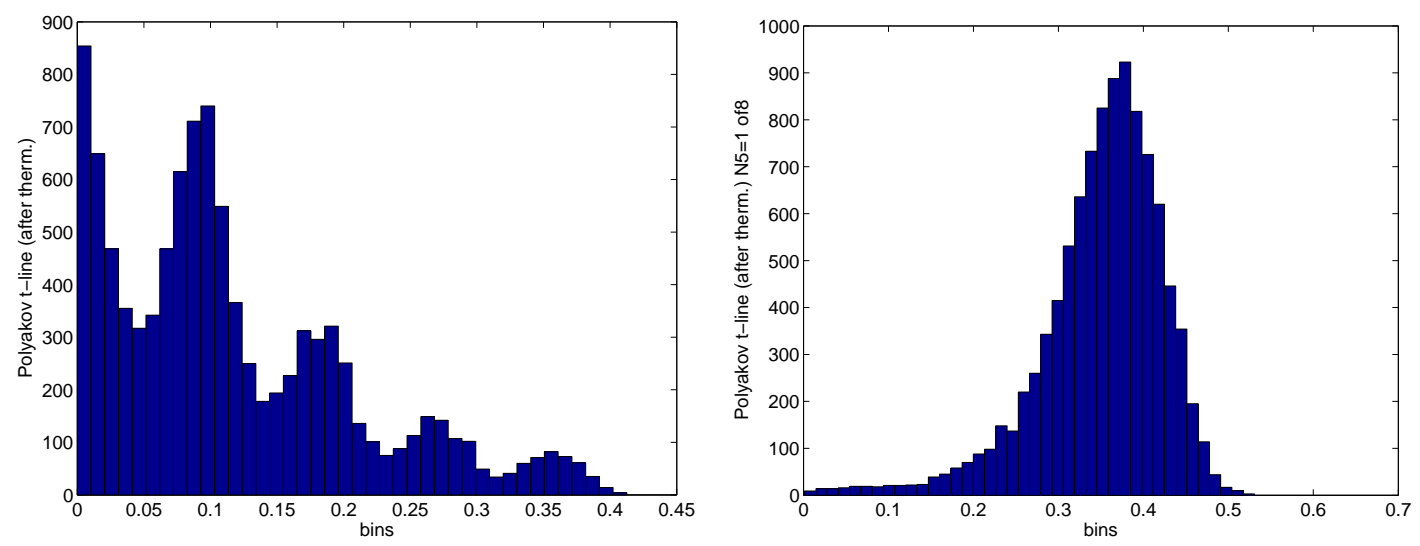

Figure 5: Histograms of the absolute value of the temporal Polyakov loop at $\beta_{4}=2.32, \beta_{5}=0.5$ and $L_{T}=4$ : taking the average over the extra dimension (left plot) or at a fixed slice (right plot) [4].

varying $L_{\mathrm{s}}=8 \ldots 24$ while keeping $L_{5}=L_{\mathrm{s}} / 2$. We measure the critical coupling $\beta_{4 c}\left(L_{\mathrm{s}}\right)$, at which the susceptibility of the temporal Polyakov loop has its maximum $\chi_{c}\left(L_{\mathrm{s}}\right)$. The data for $\beta_{4 c}$ and $\chi_{c}$ are perfectly compatible with the scaling laws

$$
\begin{aligned}
\left|\beta_{4 c}\left(L_{\mathrm{s}}\right)-\beta_{4 c}\left(L_{\mathrm{s}}=\infty\right)\right| & \sim L_{\mathrm{s}}^{-1 / v} \quad(4 \mathrm{~d} \text { Ising: } v=1 / 2), \\
\chi_{c}\left(L_{\mathrm{s}}\right) & \sim L^{\gamma / v} \quad(4 \mathrm{~d} \text { Ising: } \gamma=1),
\end{aligned}
$$

using the values of the critical exponents $v, \gamma$ of the four-dimensional Ising model. We thus confirm [9].

At $L_{T}=4$ we encountered a new phenomenon. If we average the temporal Polyakov loop over the fifth dimension and then take the absolute value multiple peaks appear in the histogram of the Monte Carlo history, see the left plot of Fig. 5. If instead we take the Polyakov loop at a fixed slice along the extra dimension, we obtain a single peak, see the right plot of Fig. 5. These observations can be interpreted if we assume that the hyperplanes along the fifth dimension are decoupling from each other. The finite size scaling analysis using the Polyakov loop at the fixed slice confirms the 

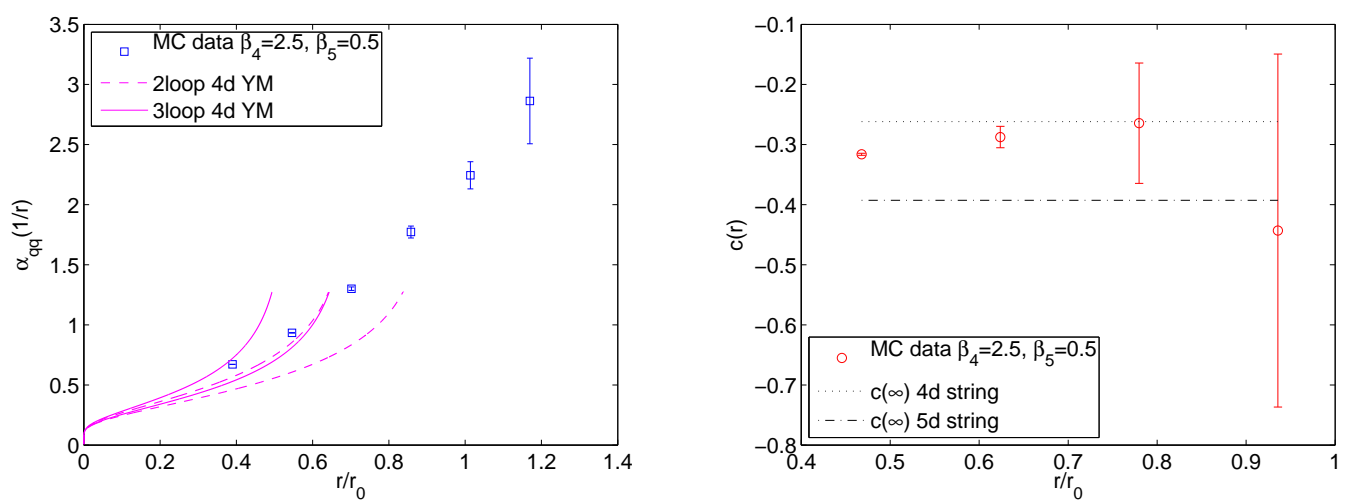

Figure 6: Shape of the static potential at $\beta_{4}=2.5, \beta_{5}=0.5$ in the confined phase. The coupling $\alpha_{\mathrm{qq}}(1 / r)=$ $4 r^{2} F(r) / 3$ (left plot) and the slope $c(r)=r^{3} F^{\prime}(r) / 2$ (right plot), where $F$ is the static force and the distance $r$ is orthogonal to the fifth dimension.

universality class of the four-dimensional Ising model also at $L_{T}=4$ [4].

\subsection{Dimensional reduction in the confined phase}

We measure the static potential in the five-dimensional confined phase in large volume. We choose $\beta_{4}=2.5$ and $\beta_{5}=0.5$, the lattice dimensions are $L_{T}=L_{\mathrm{S}}=32, L_{5}=16$ and we analyze two replica for a total of 17234 measurements. Each measurement of the Wilson loops is separated by 10 update iterations and each iteration consists of one heatbath and 16 overrelaxation sweeps through the lattice. For the time-like links we use the one-link integral [13] and for the space-like links 4 levels of spatial HYP smearing [14]. As explained in [4] we can extract the potential $V(r)$, where the distance $r$ is taken orthogonal to the fifth dimension. From the potential we determine the force $F(r)=\left\{V\left(r+a_{4}\right)-V(r)\right\} / a_{4}$ and define the renormalized couplings

$$
\alpha_{\mathrm{qq}}(1 / r)=4 r^{2} F(r) / 3 \quad \text { and } \quad c(r)=r^{3} F^{\prime}(r) / 2 .
$$

The effective bosonic string theory $[10,11]$ yields the asymptotic value $c(\infty)=-(D-2) \pi / 24$, where $D$ is the number of space-time dimensions.

The lattice spacing measured in units of the scale $r_{0}$ [12] is $r_{0} / a_{4}=6.41(21)$. In Fig. 6 we show our results for the couplings Eq. (3.3). The data for $\alpha_{\mathrm{qq}}$ are compared to perturbation theory in the four-dimensional Yang-Mills theory up to three loops and they are consistent at the two smallest distances. The slope $c(r)$ is harder to measure, it shows a trend towards the four-dimensional value of the effective string but the statistical error is already too large at $0.8 r_{0}$.

\section{Conclusions}

We have explored through Monte Carlo simulations the phase diagram of five-dimensional $\mathrm{SU}(2)$ lattice gauge theory on the torus, in the light of mean-field results for anisotropy $\gamma<1$. The phase diagram is shown in Fig. 2. The bulk phases which we identify are the confined and deconfined phase. We do not find a separate layered phase, like there is in the mean-field phase diagram Fig. 1. Nevertheless we find properties reminiscent of the layered phase, like the decoupling of the 
hyperplanes along the fifth dimension and signs of dimensional reduction in the confined phase. This could point at a localization mechanism for gauge fields, cf. [15, 16].

\section{Acknowledgments}

This work was funded by the Deutsche Forschungsgemeinschaft (DFG) under contract KN 947/1-1. In particular A. R. acknowledges full support from the DFG. The Monte Carlo simulations were carried out on the Cheops supercomputer at the RRZK computing centre of the University of Cologne and on the cluster Stromboli at the University of Wuppertal and we thank both Universities.

\section{References}

[1] M. Creutz, Confinement and the criticality of space-time, Phys. Rev. Lett. 43 (1979) 553.

[2] K. G. Wilson, Confinement of Quarks, Phys. Rev. D 10 (1974) 2445.

[3] N. Irges, F. Knechtli, Mean-Field Gauge Interactions in Five Dimensions I. The Torus, Nucl. Phys. B 822 (2009) 1, erratum: Ibid. 840 (2010) 438.

[4] F. Knechtli, M. Luz, A. Rago, On the phase structure of five-dimensional SU(2) gauge theories with anisotropic couplings, [arXiv:1110.4210].

[5] K. Yoneyama, N. Irges, F. Knechtli, The Lattice Mean-Field Approximation of Gauge-Higgs Unification on the Orbifold, these proceedings.

[6] N. Irges, F. Knechtli, A new model for confinement, Phys. Lett. B 685 (2010) 86.

[7] S. Ejiri, J. Kubo, M. Murata, A Study on the nonperturbative existence of Yang-Mills theories with large extra dimensions, Phys. Rev. D 62 (2000) 105025.

[8] P. de Forcrand, A. Kurkela, M. Panero, The phase diagram of Yang-Mills theory with a compact extra dimension, JHEP 1006 (2010) 050.

[9] B. Svetitsky, L. G. Yaffe, Critical Behavior At Finite Temperature Confinement Transitions, Nucl. Phys. B 210 (1982) 423.

[10] M. Lüscher, K. Symanzik, P. Weisz, Anomalies of the Free Loop Wave Equation in the WKB Approximation, Nucl. Phys. B 173 (1980) 365.

[11] M. Lüscher, Symmetry Breaking Aspects of the Roughening Transition in Gauge Theories, Nucl. Phys. B 180 (1981) 317.

[12] R. Sommer, A New way to set the energy scale in lattice gauge theories and its applications to the static force and $\alpha_{s}$ in SU(2) Yang-Mills theory, Nucl. Phys. B 411 (1994) 839-854.

[13] G. Parisi, R. Petronzio, F. Rapuano, A Measurement of the String Tension Near the Continuum Limit, Phys. Lett. B 128 (1983) 418.

[14] A. Hasenfratz, F. Knechtli, Flavor symmetry and the static potential with hypercubic blocking, Phys. Rev. D 64 (2001) 034504.

[15] G. R. Dvali, M. A. Shifman, Domain walls in strongly coupled theories, Phys. Lett. B 396 (1997) 64.

[16] M. Laine, H. B. Meyer, K. Rummukainen, M. Shaposhnikov, Effective gauge theories on domain walls via bulk confinement?, JHEP 0404 (2004) 027. 